Reprod. Nutr. Dévelop., 1987, 27 (1 A), 67-75.

\title{
Evolution des activités alimentaires des vaches laitières en début de lactation et liaison avec les quantités d'aliments ingérées
}

\author{
J. B. COULON, M. DOREAU, B. RÉMOND, M. JOURNET $\left({ }^{*}\right)$
}

avec la collaboration technique de B. MARQUIS $\left({ }^{*}\right)$ et A. OLLIER

Laboratoire de la Lactation,

I.N.R.A. Theix, 63122 Ceyrat, France.

Summary. Feeding behaviour of dairy cows in early lactation : Relationship with dry matter intake.

Feeding behaviour was measured in 12 multiparous dairy cows all day long during the first 5 weeks of lactation. These cows received corn silage ad libitum and concentrates representing 21 to $27 \%$ of total dry matter intake, depending on the week. During the first week of lactation, total eating and ruminating times were 364 and 426 min ; eating and ruminating rates were 39.0 and $32.5 \mathrm{~g} \mathrm{DM} / \mathrm{min}$. The feed intake increase during the first 5 weeks of lactation was accompanied by an increase in ruminating time $(+22 \%)$ and eating rate $(+26 \%)$. These two parameters were very sensitive to short-time decreases in DM intake. Animal variations of eating and ruminating rates, but not variations of total eating and ruminating times, were related to those of dry matter intake. Thus, animals which had the highest dry matter intake were those which had the highest eating and ruminating rates.

Le début de la lactation de la vache laitière se caractérise par un accroissement important de ses besoins énergétiques, azotés et minéraux. La vache alimentée à volonté y répond par une augmentation de son ingestion. Celle-ci, quoique rapide, ne suffit pas à couvrir les besoins énergétiques de l'animal fort producteur avant un mois de lactation environ, même pour des rations très digestibles et de faible encombrement (Journet et Rémond, 1976). Le délai d'adaptation du tube digestif a longtemps été considéré comme un facteur limitant de l'accroissement des quantités ingérées en début de lactation. En réalité, les variations dans le développement du tractus digestif accompagnent celles des quantités ingérées (Fell et Weekes, 1975), sans qu'il y ait d'effet propre du stade physiologique (Doreau et al., 1985b). Par ailleurs, l'accroissement important du remplissage du rumen s'effectue dès la première semaine de lactation (Doreau et al., 1985a).

Pour accroître son ingestion, l'animal doit manger plus longtemps et/ou plus vite, et il est possible que ces adaptations du comportement alimentaire en début

(*) Adresse actuelle: Station de Recherches sur la Vache Laitière, I.N.R.A. St-Gilles, 35590 L'Hermitage, France. 
de lactation limitent en partie l'accroissement des quantités ingérées. Dans un essai précédent, Doreau et Rémond (1982) ont montré que la vache laitière était capable d'augmenter très fortement sa durée d'ingestion d'une ration très ingestible à base d'ensilage de maïs, en particulier grâce à une plus grande durée des repas qui suivent la distribution des aliments. On ne connaît pas l'existence éventuelle de valeurs maximales des différents paramètres des activités alimentaires, ni les conditions déterminant leurs variations. Leur étude fait l'objet de cet essai qui se place dans le cadre d'une expérimentation plus large sur la capacité d'ingestion des vaches laitières en début de lactation (Coulon et al., 1984a et b) et de leur métabolisme énergétique (Coulon et al., 1985). Nous avons cherché à analyser les variations journalières et individuelles des activités alimentaires, en examinant les liaisons entre les différents paramètres mesurés et avec les quantités ingérées et les caractéristiques des animaux.

\section{Matériel et méthodes.}

Animaux et alimentation. - Douze vaches de type Pie-Noir, en stabulation entravée, en $2^{\mathrm{e}}$ lactation et plus, et d'un poids moyen après le vêlage de $555 \mathrm{~kg}$ ont été utilisées. En fin de gestation (deux dernières semaines), elles ont reçu $2 \mathrm{~kg}$ de matière sèche (MS) d'ensilage de maïs par $100 \mathrm{~kg}$ de poids vif, $1 \mathrm{~kg}$ de tourteau tanné et $150 \mathrm{~g}$ d'urée. En début de lactation (5 premières semaines), l'ensilage de maïs a été distribué à volonté. Les quantités d'aliments concentrés offerts ont été, à une semaine donnée, les mêmes pour tous les animaux. Elles sont passées de $3,0 \mathrm{~kg} / \mathrm{j}$ en $1^{\mathrm{re}}$ semaine à $5,5 \mathrm{~kg} / \mathrm{j}$ en $5^{\mathrm{e}}$ semaine. De cette façon, en $5^{\mathrm{e}}$ semaine de lactation, la ration totale satisfaisait les besoins énergétiques d'entretien plus ceux nécessaires à la production de $32 \mathrm{~kg}$ de lait à $4 \%$ de matières grasses qui était la production maximale moyenne attendue d'après la lactation précédente. La composition de l'aliment concentré a été adaptée chaque semaine à chaque animal en faisant varier la proportion de tourteau tanné de manière à couvrir les besoins azotés individuels dès la $1^{\text {re }}$ semaine de lactation. La quantité d'aliment concentré a représenté respectivement $21,24,24,26$ et $27 \%$ de ra ration totale au cours des 5 premières semaines de lactation. L'ensilage de maïs, l'urée $(200 \mathrm{~g} / \mathrm{j})$, le complément minéral $(300 \mathrm{~g} / \mathrm{j})$ et les aliments concentrés ont été mélangés et distribués individuellement en 2 fois, en quantités égales à $8 \mathrm{~h} 30$ et 17 h 30.

Mesures. - Les quantités d'aliments offertes et refusées et la production laitière ont été mesurées tous les jours. Les animaux ont été pesés 3 fois au cours de la $1^{\text {re }}$ semaine de lactation et une fois par semaine ensuite.

Les activités alimentaires ont été enregistrées en continu à partir du $2^{\mathrm{e}}$ ou $3^{e}$ jour de lactation et jusqu'à la fin de la $5^{e}$ semaine de lactation. Les mouvements de la mâchoire ont été transmis par un ballonnet fixé en position sousmaxillaire à un capteur pneumatique relié à un enregistreur graphique (Ruckebusch, 1963). Par convention, on a appelé repas toute période d'ingestion de plus de $5 \mathrm{~min}$, éventuellement entrecoupée d'arrêts de moins de $5 \mathrm{~min}$. Les 2 repas suivant les distributions d'aliments, qui sont les plus longs, ont été appelés grands 
repas et les autres, petits repas. Compte tenu des quelques données manquantes liées à des incidents techniques, l'ensemble des résultats qui suivent porte sur 386 observations journalières ( 29 à 35 jours de mesure sur 12 animaux).

\section{Résultats.}

\section{Evolution des paramètres du comportement alimentaire.}

Alors que les quantités ingérées par les 12 vaches ont augmenté en moyenne de $5,5 \mathrm{~kg}$ de MS (soit de $43 \%$ ) entre la $1^{\mathrm{re}}$ et la $6^{\mathrm{e}}$ semaine de lactation, la durée d'ingestion a peu varié au cours de cette période (tabl. 1) : elle n'a augmenté que de 30 min $(+9 \%)$ entre la $1^{\text {re }}$ et la $5^{\theta}$ semaine où elle a été maximale. C'est donc la vitesse d'ingestion qui a augmenté au cours de l'essai $\left(+26 \%\right.$ entre la $1^{\text {re }}$ et la $4^{e}$ semaine). Au cours de cette période (semaines 1 à 4) la durée de rumination a par contre augmenté de $1 \mathrm{~h} 30(+22 \%)$ et la vitesse de rumination de $3,6 \mathrm{~g}$ $\mathrm{MS} / \min (+11 \%)$. L'augmentation de la durée de rumination est due essentiellement à l'augmentation de la durée de chaque période de rumination $1+16 \%$ entre la $1^{\text {re }}$ et la $4^{e}$ semainel, le nombre de périodes n'ayant pratiquement pas varié, de même que le nombre de repas (tabl. 1). La durée du grand repas du matin a augmenté d'environ 20 min entre la première et la $5^{e}$ semaine de lactation, mais pas celle du grand repas du soir, peut-être en raison d'un état de réplétion plus prononcé du rumen en cours de journée qu'après un jeûne relatif nocturne.

De même que pour les quantités ingérées et la production laitière (cf. Coulon et al., 1984a), l'essentiel de ces augmentations s'est réalisé au cours des 15 premiers jours de lactation (fig. 1), mais de manière très variable d'un animal à l'autre: ainsi, les différences entre la $1^{\text {re }}$ semaine et la semaine où la valeur de l'activité alimentaire correspondante a été maximale ou minimale, ont varié selon les vaches de -110 et -58 min à respectivement +190 et $+250 \mathrm{~min}$, pour les durées d'ingestion et de rumination et de $-8,3$ et $-13,3 \mathrm{~g} \mathrm{MS} / \mathrm{min}$ à respecti-

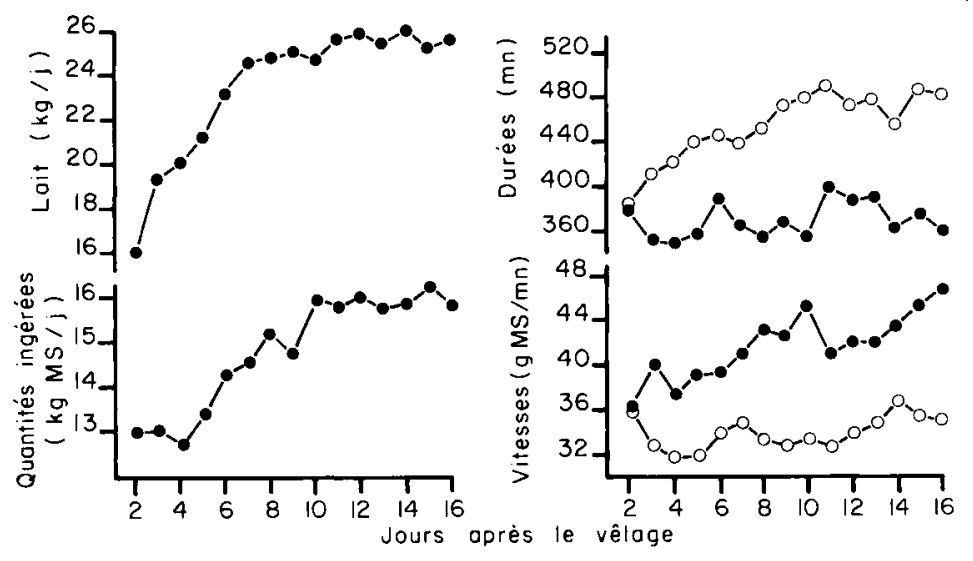

FIG. 1. - Evolution de la production laitière, des quantités ingérées et des activités alimentaires (- Ingestion, O Rumination) au début de la lactation (moyenne des 12 vaches sauf les jours 2 ( 5 vaches) et 3 (9 vaches)). 


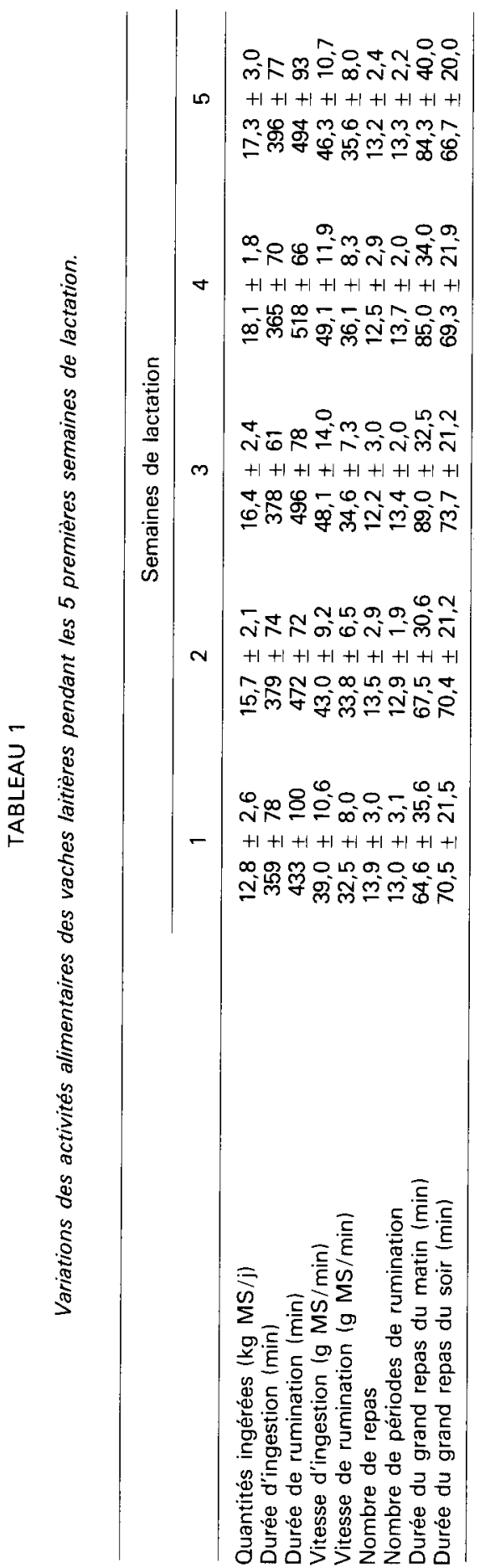


vement $+32,0$ et $+16,7 \mathrm{~g} \mathrm{MS} / \mathrm{min}$ pour les vitesses d'ingestion et de rumination. Ces différences individuelles sont beaucoup plus importantes que celles des accroissements des quantités ingérées (tabl. 2). D'autre part, elles sont d'autant plus importantes que les valeurs initiales (semaine 1) de l'activité alimentaire correspondante sont plus faibles $(P<0,05)$. Ainsi, plus les animaux ingèrent ou ruminent longtemps en $1^{\text {re }}$ semaine, moins ils augmentent leur durée d'ingestion ou de rumination par la suite, et donc plus ils augmentent leur vitesse correspondante. Par contre, les variations d'activités alimentaires ne sont pas ou peu liées aux quantités ingérées en $1^{\text {re }}$ semaine ou aux accroissements maximaux des quantités ingérées. De même, il n'y a pas eu de liaison significative entre l'augmentation de la durée d'ingestion et celle de la durée de rumination $(R=0,31$, $P>0,05)$.

\section{TABLEAU 2}

Valeurs hebdomadaires initiales (semaine $1=\mathrm{S} 1$ ) et extrêmes (SM) des activités alimentaires (moyenne et écart-type inter-vaches).

\begin{tabular}{lccc}
\hline & $\mathrm{S} 1$ & $\mathrm{SM}$ & $\mathrm{D}=\mathrm{SM}-\mathrm{S} 1$ \\
\hline Quantités ingérées (kg MS) & $12,8 \pm 2,1$ & $19,2 \pm 1,9$ & $6,4 \pm 2,5$ \\
Durée d'ingestion (min) & $359 \pm 49$ & $393 \pm 72$ & $34 \pm 87$ \\
Durée de rumination (min) & $433 \pm 80$ & $524 \pm 69$ & $92 \pm 85$ \\
Vitesse d'ingestion (g MS/min) & $39,0 \pm 8,2$ & $50,9 \pm 10,9$ & $11,9 \pm 11,3$ \\
Vitesse de rumination $(\mathrm{g} \mathrm{MS} / \mathrm{min})$ & $32,5 \pm 5,8$ & $36,0 \pm 6,6$ & $3,5 \pm 7,2$ \\
\hline
\end{tabular}

Evolution des paramètres du comportement au moment de chutes d'ingestion.

Au cours des 5 premières semaines de lactation, nous avons enregistré 15 chutes d'ingestion (mises en évidence en comparant la courbe individuelle d'évolution des quantités ingérées à la courbe moyenne de 42 vaches ; cf. Coulon et al., 1984b), d'une amplitude moyenne de $3,5 \mathrm{~kg}$ de MS, chez les 12 vaches de l'essai. Au moment de ces chutes, la durée d'ingestion et la vitesse de rumination ont été peu modifiées. Par contre, chez pratiquement tous les animaux, la vitesse d'ingestion a diminué en movenne d'environ $8 \mathrm{~g} \mathrm{MS} / \mathrm{min}$ et la durée de rumination a diminué de près d'une heure (fig. 2), soit des variations d'environ $15 \%$.

Liaisons entre les différences individuelles des paramètres du comportement et des quantités ingérées.

Les durées d'ingestion et de rumination moyennes individuelles des 5 premières semaines de lactation n'ont pas été liées entre elles et ont été indépendantes des quantités ingérées ; elles ont donc été liées aux vitesses correspondantes. Les animaux qui mangent et qui ruminent le plus longtemps sont donc ceux qui mangent et qui ruminent le moins vite. En revanche, les vitesses d'ingestion et de rumination ont été fortement liées aux quantités ingérées $(P<0,01)$ : elles augmentent respectivement de 3,2 et $2,1 \mathrm{~g} \mathrm{MS} / \mathrm{min}$ lorsque les quantités ingérées augmentent de $1 \mathrm{~kg}$ de $M S$; les animaux qui ingèrent le plus ingèrent et ruminent donc aussi le plus vite. 


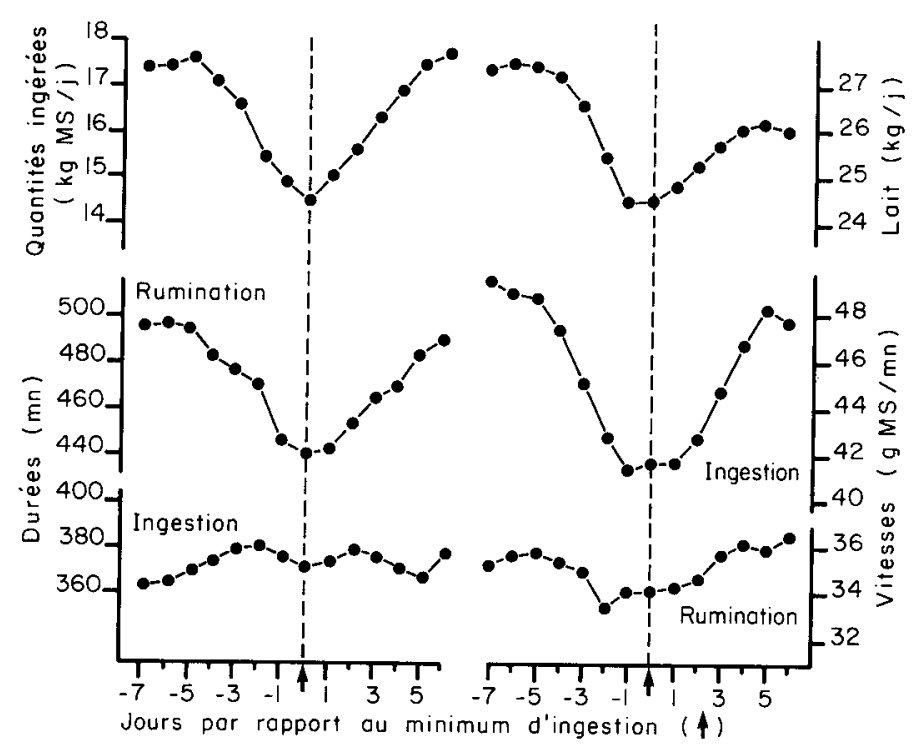

FIG. 2. - Evolution journalière comparée des quantités ingérées et de certains paramètres du comportement (valeurs exprimées en moyennes mobiles sur 5 jours) au moment des chutes d'ingestion (moyenne des 15 chutes au moment desquelles le comportement alimentaire était enregistrél.

Les durées d'ingestion et de rumination n'ont pas été significativement liées au poids vif ou au potentiel laitier (estimé par la production des $4^{\mathrm{e}}, 5^{\mathrm{a}}$ et $6^{\mathrm{e}}$ jour de lactation) des animaux. En revanche, la vitesse de rumination a été d'autant plus élevée que le poids vif était plus important $(P<0,01)$, certainement parce que les animaux les plus lourds avaient la possibilité de traiter plus de matière sèche par coup de mâchoire (Dulphy, 1971). La vitesse d'ingestion n'a pas été liée au poids vif, mais elle a été d'autant plus élevée que le potentiel de production laitière était plus important $(P<0,05)$.

Les variations d'un jour à l'autre de la durée d'ingestion au cours des 35 premiers jours de lactation ne rendent compte que d'une faible partie des variations des quantités ingérées $(r=0,41)$. D'ailleurs, la liaison $n^{\prime}$ est significative que chez 2 des 12 animaux. Par contre, la durée de rumination, indépendante de la durée d'ingestion, augmente fortement avec les quantités ingérées, en moyenne de 19 min par $\mathrm{kg}$ de MS ingérée (12 à 30 selon les animaux) et la liaison entre ces 2 paramètres est très étroite $(r=0,88)$. Inversement, la vitesse de rumination, bien que significativement liée aux quantités ingérées $(r=0,63)$, augmente beaucoup moins vite que la vitesse d'ingestion lorsque les quantités ingérées augmentent de $1 \mathrm{~kg}$ de MS $(6 \mathrm{~g} \mathrm{MS} / \mathrm{min}$ contre $22 \mathrm{~g} \mathrm{MS} / \mathrm{min})$.

L'augmentation des quantités ingérées en début de lactation ne s'est jamais accompagnée à la fois d'une augmentation importante des durées (d'ingestion ou de rumination) et des vitesses correspondantes. On peut en fait distinguer 3 groupes d'animaux (fig. 3) : ceux qui augmentent uniquement leurs durées d'activité alimentaire (ingestion et rumination) $(n=2)$, ceux qui augmentent uniquement 
leurs vitesses d'activité alimentaire ( $n=4)$, et ceux qui augmentent à la fois leur vitesse d'ingestion et leur durée de rumination $(n=6)$. Ces 3 groupes se différencient plus par leur écart de niveau initial d'activité alimentaire (environ 45 min d'écart de durée d'ingestion et de rumination entre le groupe 1 et les groupes 2 et 3 en première semaine de lactation, en faveur des groupes 2 et 3) que par leur poids vif (respectivement 575,574 et $540 \mathrm{~kg}$ au vêlage pour les 3 groupes), leur potentiel de production (respectivement $19,9,24,4$ et $20,8 \mathrm{~kg} / \mathrm{j}$ ) ou leur niveau d'ingestion (respectivement $13,2,13,5$ et $12,4 \mathrm{~kg} \mathrm{MS} / \mathrm{j}$ au cours de la première semaine de lactation). Il est cependant étonnant que les animaux du groupe 3 , bien qu'ayant une durée de rumination initiale (semaine 1) élevée (436 min), l'aient fortement augmenté par la suite.

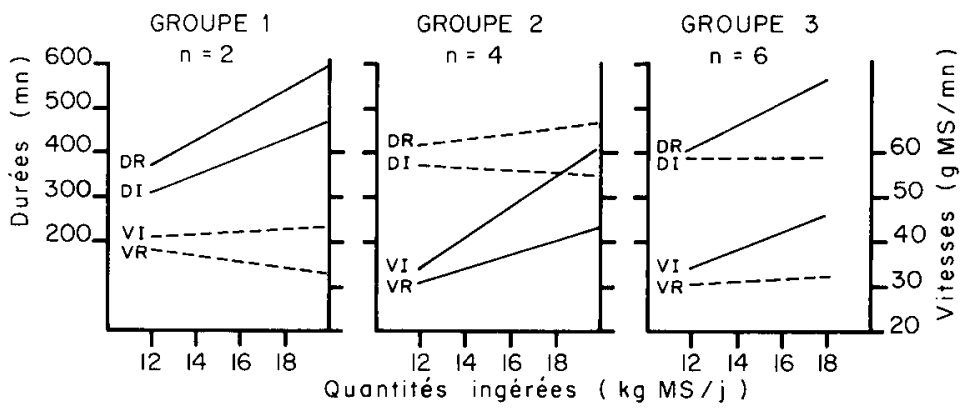

FIG. 3. - Liaisons entre les paramètres du comportement alimentaire (DI : durée d'ingestion ; DR : durée de rumination ; $\mathrm{VI}$ : vitesse d'ingestion ; VR : vitesse de rumination) et les quantités ingérées, entre le $2^{e}$ et le $35^{e}$ jour de lactation ( $n=33$ points par régression).

Les pentes des droites de régression en pointillés ne sont pas significativement différentes de 0 au seuil de $1 \%$.

\section{Discussion.}

Dans cet essai, contrairement à ce qui est couramment observé (Campling, 1966 ; Journet et Rémond, 1976 ; Doreau et Rémond, 1982), les animaux ont accru leur ingestion en augmentant leur vitesse d'ingestion et non leur durée, ce qui correspond aux résultats de Faverdin (1985), obtenus avec un temps d'accès limité à l'aliment. Ce résultat doit être relié au niveau relativement élevé au vêlage de la durée d'ingestion par rapport à ceux des auteurs précédents, même avec un régime voisin (Doreau et Rémond, 1982) ; d'ailleurs, les quelques animaux qui présentaient dans notre essai une durée d'ingestion faible au vêlage l'ont effectivement augmenté par la suite. II est donc vraisemblable que des caractéristiques individuelles doivent influencer la durée d'ingestion des animaux (Dulphy, 1971).

II est également possible que l'augmentation de la vitesse d'ingestion (plutôt que de la durée), qui est considérée comme un bon indicateur de l'état de faim de l'animal (Hesselbarth, 1954), traduise un accroissement de cet état de faim au cours des premières semaines de lactation (Faverdin, 1985) et ne soit pas lié au déficit énergétique de l'animal qui diminue au cours de cette période. Ceci est à relier au fait que les animaux les plus forts producteurs ont ingéré le plus vite, non pas parce qu'ils recevaient plus d'aliment concentré étant donné notre protocole 
expérimental, mais peut-être parce qu'ils avaient plus d'appétit, en raison de leurs besoins plus élevés.

Dans notre essai, il semble d'autre part peu probable que l'accroissement de la vitesse d'ingestion soit dû à l'augmentation du pourcentage de concentré dans la ration : celui-ci a en effet peu varié et la ration était offerte en mélange.

L'augmentation de la vitesse d'ingestion en début de lactation est peut-être à mettre en cause dans les modifications de la digestion dans le rumen souvent observées à cette période (Everson et al., 1976; Doreau et Rémond, 1982). Elle pourrait entraîner une baisse de la digestibilité de la matière sèche en affectant la réduction des aliments en fines particules lors de la mastication initiale, d'autant plus que la vitesse de mastication mérycique a plutôt tendance à augmenter. II est aussi possible que les liaisons significatives entre les différences individuelles de vitesse d'ingestion ou de rumination et les quantités ingérées expliquent des différences de digestibilité de la matière sèche entre animaux de niveau d'ingestion diffèrent (Tyrrell et Moe, 1975). Les animaux qui consomment le plus de matière sèche sont en effet ceux qui ingèrent et ruminent le plus vite, comme l'avaient déjà observé Kerbaa (1969) chez des vaches laitières et Dulphy (1971) chez des moutons. Mais il est difficile de savoir si ces variations du comportement sont la cause ou la conséquence des variations des quantités ingérées.

L'accroissement du niveau d'ingestion en début de lactation s'est accompagné d'une augmentation de la durée de rumination plus que de la vitesse. Ceci correspond aux observations faites avec des régimes à base d'ensilage de maïs (Journet et Rémond, 1976 ; Doreau et Rémond, 1982) où les durées initiales de rumination, comme dans notre essai, étaient faibles ; lorsque c'est l'inverse (régimes à base de foin), c'est la vitesse de rumination qui augmente en début de lactation (Campling, 1966 ; Journet et Rémond, 1976). Cette augmentation de la durée de rumination a été étroitement liée à celle des quantités ingérées, tout comme chez Welch et Smith (1969) et Dulphy (1971). Elle s'est réalisée grâce à une augmentation de la durée de chaque période et non de leur nombre, qui ne dépend pas des quantités ingérées (Freer et al., 1962).

En définitive, il semble donc qu'en début de lactation les vaches laitières augmentent leurs quantités ingérées en accroissant soit leurs durées d'activité alimentaire (ingestion et/ou rumination) lorsqu'elles sont faibles, soit leurs vitesses lorsque les durées correspondantes sont élevées. Mais elles augmentent rarement à la fois la durée et la vitesse d'une même activité. Il est possible qu'il existe un certain équilibre entre ces 2 paramètres : Welch et Smith (1969) ont ainsi mis en évidence une relation curvilinéaire entre la durée de rumination et les quantités ingérées : lorsque celles-ci atteignent une certaine valeur, la durée de rumination n'augmente plus, c'est donc la vitesse qui varie alors. II subsiste cependant d'importantes différences individuelles dans l'évolution de ces paramètres dont les causes (digestives, métaboliques, hormonales - cf. Dulphy et Faverdin, 1987) restent à définir. 


\section{Références}

CAMPLING R. C., 1966. A preliminary study of the effect of pregnancy and of lactation on the voluntary intake of food by cows. Br. J. Nutr., 20, 25-39.

COULON J. B., DOREAU M., RÉMOND B., JOURNET M., 1984a. Capacité d'ingestion des vaches laitières en début de lactation. 1. Evolution et liaison avec certains paramètres zootechniques. Conséquences sur la production laitière. Bull. Tech. CRZV Theix, I.N.R.A., 55, 33-42.

COULON J. B., DOREAU M., RÉMOND B., JOURNET M., 1984b. Capacité d'ingestion des vaches laitières en début de lactation. 2. Les anomalies de l'ingestion : Liaisons avec l'état sanitaire et quelques paramètres du métabolisme énergétique et de la digestion. Bull. Tech. CRZV Theix, I.N.R.A., 55, 43-51.

COULON J. B., REMOND B., DOREAU M., JOURNET M., 1985. Evolution de différents paramètres sanguins du métabolisme énergétique chez la vache laitière en début de lactation. Ann. Rech. vét., 16, 185-193.

DOREAU M., RÉMOND B., 1982. Comportement alimentaire et utilisation digestive d'une ration de composition constante chez la vache laitière en fin de gestation et début de lactation. Reprod. Nutr. Dévelop., 22, 207-324.

DOREAU M., DELACROIX A., RÉMOND B., 1985a. Liaisons entre la digestion et le stade physiologique chez la vache laitière : rôle du temps de transit. Reprod. Nutr. Dévelop., 25, 779 .

DOREAU M., ROBELIN J., LESTRADE A., 1985b. Effects of physiological state and body fatness on digestive-tract weight and composition in the dairy cow. Livest. Prod. Sci., 12, 379-385.

DULPHY J. P., 1971. Influence du poids vif et du niveau d'ingestion sur le comportement alimentaire et mérycique du mouton. Ann. Zootech., 20, 447-486.

DULPHY J. P., FAVERDIN P., 1987. La prise alimentaire chez les ruminants: volume et modifications digestives et métaboliques qui l'accompagnent. Reprod. Nutr. Dévelop., 27. 129-155.

EVERSON R. A., JORGENSEN N. A., CROWLEY J. W., JENSEL E. L., BARRINGTON J. P., 1976. Input output of dairy cows fed a complete ration of a constant or variable forage to grain ratio. J. Dairy Sci., 59, 1776-1787.

FAVERDIN P., 1985. Régulation de l'ingestion des vaches laitières en début de lactation. Th. Doct.-Ingén., INA-PG, $113 \mathrm{p}$.

FELL B. F., WEEKES R. E. C., 1975. Food intake as a mediator of adaptation in the ruminal epithelium. In J. W. Mc DONALD and A. C. I. WARNER, Digestion and metabolism in the ruminant. 101-118. (Armidale, NSW, Univ. New England Publ. Unit).

FREER M., CAMPLING C., BALCH C. C., 1962. 4. Behaviour and reticular motility of cows receiving diets of hay, oat straw with urea. Br. J. Nutr., 16, 279-295.

HESSELBARTH K., 1954. Untersuchungen über Fresslust, Futteraufnahmevermogen und Futterverwertung bei Milchkuhen. Archiv. Tierernahrg., 4, 145-195.

JOURNET M., RÉMOND B., 1976. Physiological factors affecting the voluntary intake of feed by cows : a review. Livest. Prod. Sci., 3, 129-146.

KERBAA A., 1969. Etude de quelques facteurs de variations du comportement alimentaire et mérycique des ruminants. Th. Doct. Univ. Clermont-Ferrand, $104 \mathrm{p}$.

RUCKEBUSCH Y., 1963. Recherches sur la régulation centrale du comportement alimentaire chez les ruminants. Th. Doct. Sci. Univ., Lyon, $213 \mathrm{p}$.

TYRRELL H. F., MOE P. W., 1975. Effect of intake on digestive efficiency. J. Dairy Sci., 58, 1151-1163.

WELCH J. G., SMITH A. M. 1969. Effect of varying amounts of forage intake on rumination. J. anim. Sci., 28, 827-835. 